\title{
Spinal Engine \& Waist Power from Taijiquan Viewpoint
}

\begin{abstract}
The myriad muscle actions of body segments have to be harmonized to produce motion of unified momentum underlying maximal waist power. Critical is the harmony of the torques at the waist-groin region, namely, that at the hip-joints of the pelvic movements and the spinal torsion. The practice solution to cultivate this harmony is forged by Taijiquan's principle of centrality that sorts out the multitudes of muscles in their functional roles of core-stabilization, postural control, and propulsion, as well as breathing, and allows them to balance and align accordingly. As it turns out, the centrality principle of yin-yang theory mirrors the SIJ hub in the transfer of forces between the upper and lower body through the three levers, the spine and the legs via the pelvic platform. All these must pay heed to the spinal engine.
\end{abstract}

\section{Introduction}

The trunk's rotational motion is most crucial in the art of body motion. The body's core strength in sports and martial arts comes from the waist-groin, which relies critically on the trunk's rotation. And the role of the spinal engine is critical in the trunk's rotation. The spinal engine refers to the mechanism that introduces torsion in the spine by the lateral bending of the vertebral column due to the spinal curvatures. The pelvis is always tilting one way or another in body motion, causing the spinal column to flex, and thus inducing spinal torsion. The spinal engine is always running in body motion.

The trunk's rotation is primarily produced by the torques of muscle forces, which introduces torsion in the spine. This is independent of the spinal torsion produced by the spinal engine. The trunk is also riding on the pelvic platform, and the rotation at the waist involves the play of the pelvis, which links directly to the spinal engine. To produce the waist power necessary at high-level performances, the rotational motion of the trunk and the internal motion at the hipjoints, must harmonize with the spinal engine. Indeed, slight errors in managing the spinal engine and the pelvic platform can amplify to degrade waist-power output significantly, notably in the golf-range.

Developing waist power requires the discipline of the body's many segments, each powered by its own muscles, to move in unison to harmonize momentum. Unfortunately, the muscles do not respond to commands in such cooperation. The muscles underlying the movements of say a punch action, are not dedicated in their functions, but play multiple roles, which may be competing-the muscles have to attend to the stabilization, postural control, and balance of the body, as well as breathing. On top of that, the body is stubborn in its behavioral responses of muscles that often end up undermining the intended actions. Unless these issues are addressed and resolved at the basic level, training cannot improve the waistpower output significantly. But the task is illusive. That is why it is not easy for weekend golfers to increase significantly their average drive.

This paper looks at how Taijiquan provides a practical solution that comprehensively resolves the complexity of the lever system of the many segments, the effects of the spinal engine and the responses of the neuromuscular system. The diverse factors are pulled together
Journal of

Integrative Medicine \& Therapy

\section{C.P. Ong*}

Tai Chi Instructor/Author, USA

*Address for Correspondence

C P Ong, Ph.D, Tai Chi Instructor/Author, USA, E-mail: cp11ong@gmail.com

Submission: 3 August, 2017

Accepted: 2 November, 2017

Published: 12 November, 2017

Copyright: (๑) 2017 Ong CP. This is an open access article distributed under the Creative Commons Attribution License, which permits unrestricted use, distribution, and reproduction in any medium, provided the original work is properly cited.

by a centrality principle of Taijiquan, which treats as paramount the core-stabilization role of muscles, thus keeping the integrity of the body structure intact under all situations. The unorthodox slowmotion practice is directed at cultivating this centrality principle, which turns out to be an assertion of the hub principle of the sacraliliac joint (SIJ): SIJ is the 'hub' of forces transferred from the trunk to the ground and vice versa. There is a larger pay-off. Studying how Taijiquan deals with these issues in the context of generating strength, shines the light of physics and physiology on the esoteric yin-yang framework, thus giving us a more concrete understanding of the traditional theories.

\section{The Spinal Engine}

Torsion is produced when a rod is twisted by applying torque. But

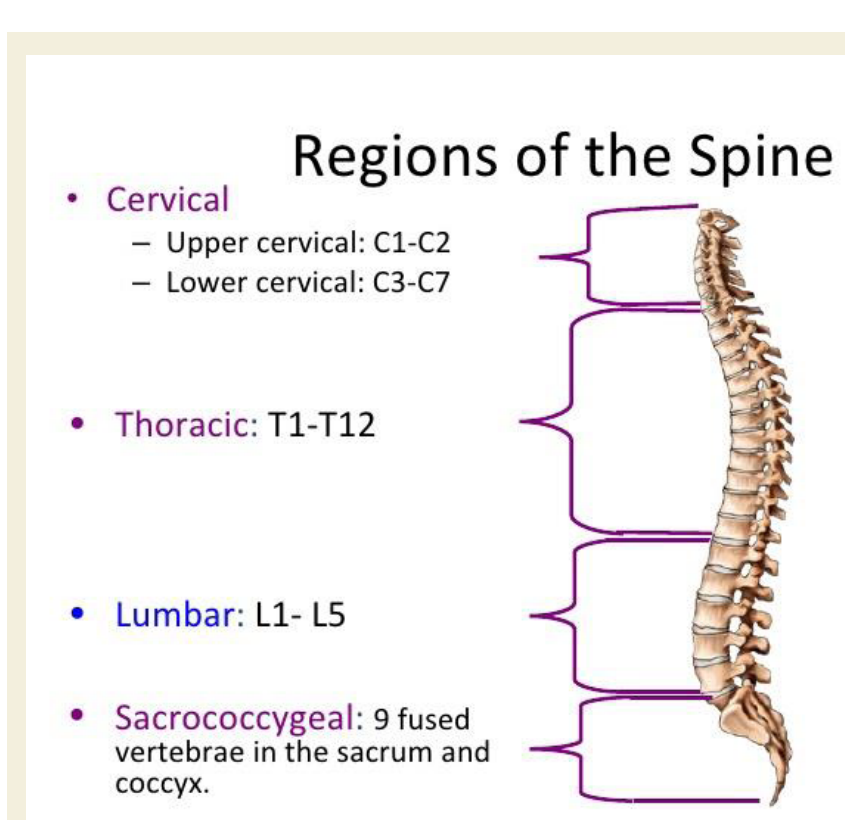

Figure 1: Spinal curvatures. 


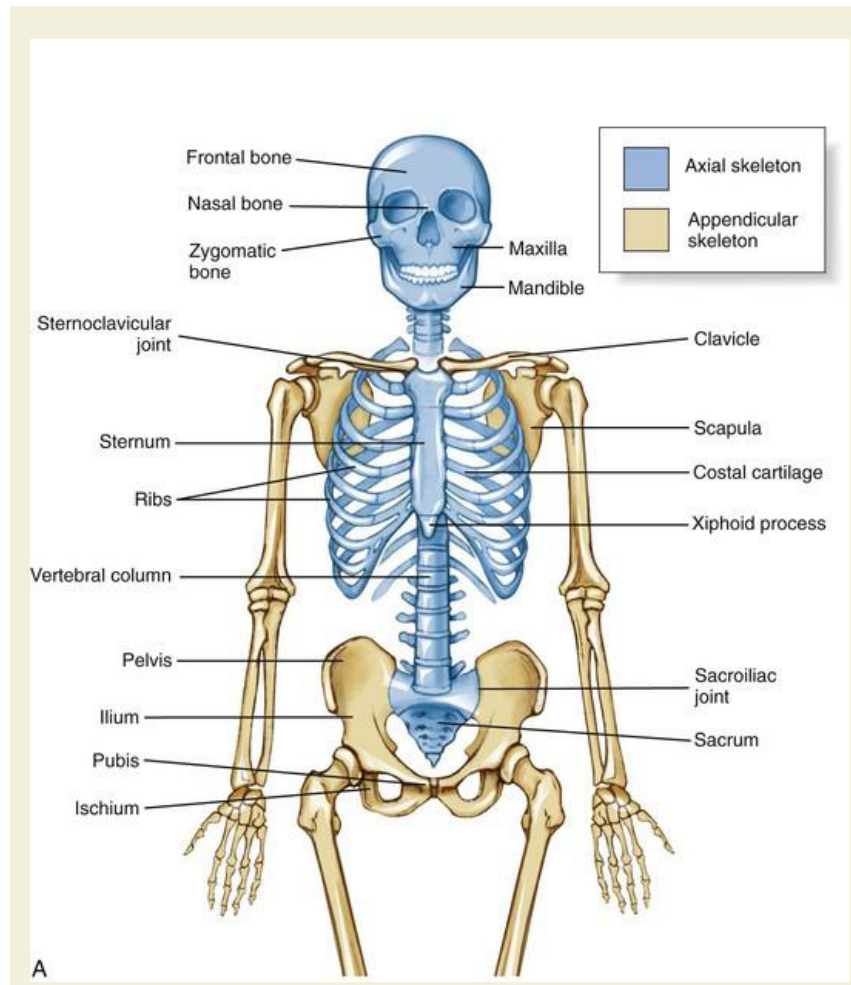

Figure 2: Skeletal frame.

not all torsion is born of a twist. We can produce torsion in a rod by bending only, without twisting. Bend a coat-hanger wire in one plane (without twisting), and then bend the curved wire in another plane. The second bending moves the wire out of its plane and introduces torsion in the wire. So whenever a curved rod is flexed laterally (bent transversely to its plane of curvature), an axial torsion is produced. When we turn our torso, muscle actions produce a torque creating torsion in the spine. However, because of our spinal curvatures torsion is also produced whenever the spine bends out of the sagittal plane (Figure 1). The mechanism that produces the torsion in the spine by virtue of its curvatures and bending motions is called the spinal engine. This axial torsion of the spinal engine is often caused by the pelvis tilting, which results in the lateral bending of the spine.

This mode of conversion between linear bending motions and axial torsion does not appear in the designs of locomotive engineering, which rely on cogs, gears and shaft. However, these mechanical devices are not available to the skeletal structures of animals. The vertebrates rely on bending motions and curvatures to produce torsion in their locomotion. This principle is very evident in the locomotion of lizards or reptiles in the lateral flexion and extension of the body (https:// giphy.com/gifs/ZIuyq5yzyvfRC).

Gracovetsky (1985) who formalized the Spinal Engine Theory credits Lovett (1898) and Farfan (1975) as having first observed the coupling between axial torsion (rotation) and lateral bending (linear) due to the spinal curvatures [1]. The Spinal Engine Theory has three components: curvature, lateral bending, and axial torsion or rotation. The coupling theory states that the combination of any two components produces the third. Our gait relies on the axial torsion produced by the combination of lateral flexion and lordosis. Lateral bending motions are often necessary to balance in juking and dodging in sports, which are produced by the combination of lordosis and axial torsion.

In the 1968 Mexico Olympics, Dick Fosbury approached the jump on the left side and propelled his body up on his right foot. His torso flexed laterally on the left as it powerfully rotated to the right. The combination of flexion and torsion increased his lordosis, enabling him to clear the bar on his back, with his center of mass below it. Dick took gold and set a world record in the showcase debut jump, which has come to be known as the Fosbury Flop. We next review briefly the anatomy of the vertebral column to see better the spinal engine in our gait, dance, and sports to bring more awareness. At the same time, the review serves to point out the challenges posed by the spinal torsion in generating body strength, and thus its criticality in sports and martial arts training.

\section{The Unsoundness of the Skeletal Structure}

The most striking feature of the human skeletal frame is its structural unsoundness-the prominent ribcage hanging on the vertebral column supported precariously at the sacrum, which is joined onto the pelvic girdle (see Figure 2). The skeletal frame is very contrary to the engineering designs that are heavily grounded on base structures for support. The spinal column consisting of vertebrae, one on top of another, and resting on the pelvic girdle at the bottom, cannot be more unsound and unstable-it requires a costly process of constant monitoring and muscle actions to maintain the integrity of the column and to keep it balanced.

To compound the challenge, the muscles that work the complex of bone levers are confined within the skin to stay close to the bones. This means that the muscles have to pull the bones at attachments close to the hinge with short moment arm. For example, the biceps are attached to the radius bone close to the elbow (see Figure 3). Try raising a flag pole with the rope attached near the base, and you can imagine the enormous force the biceps have to exert to flex the arm, which add a huge burden to the muscles. Such an engineering design, which requires continual computational and energy resources on demand to function, would seem unfeasible. Unwieldy as nature's engineering blueprints may be, dressed in muscles and equipped

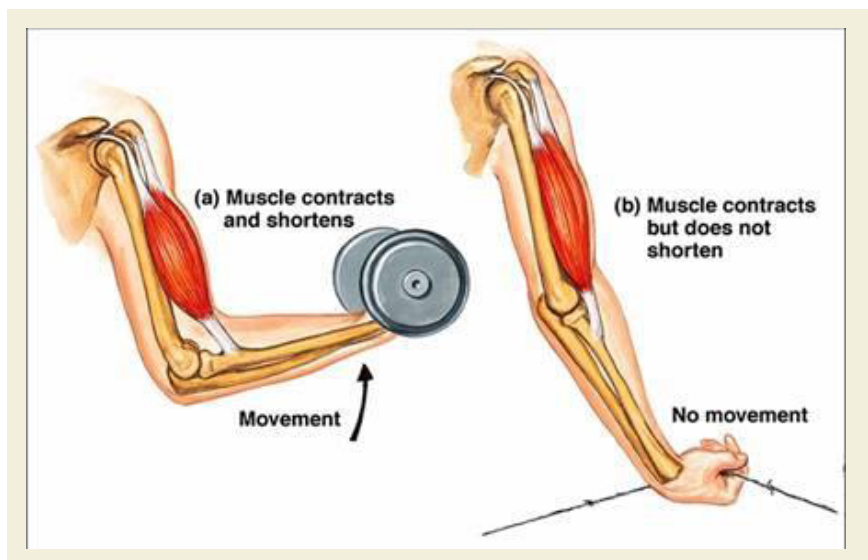

Figure 3: The biceps attach to the radius bone close to the elbow. 


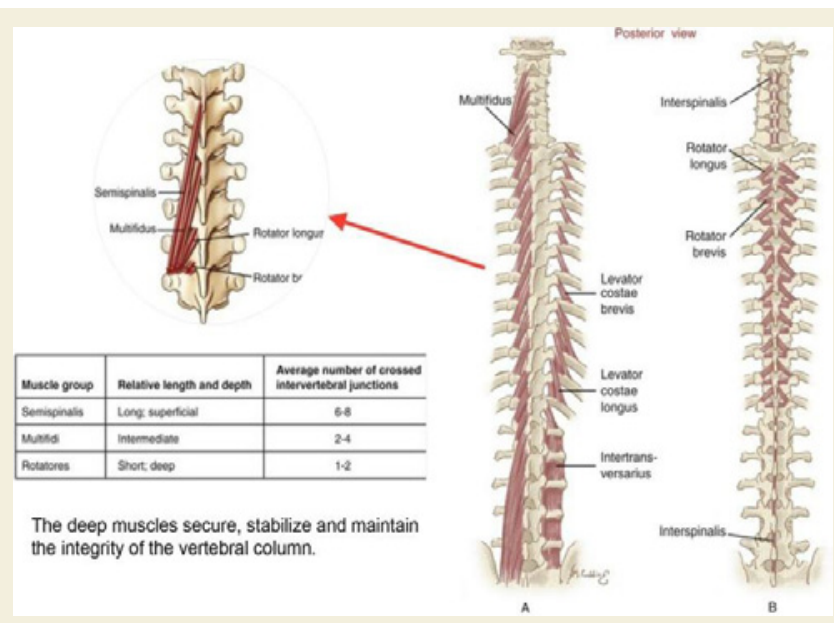

Figure 4: Deep muscles of the vertebral column.

with the neural networks, they give us a human machine that is incomparable in the range of functionality and in the fineness and precision of control.

Enormous resources of muscles operate the complex of bone lever systems. The countless muscle fibers are organized into individual motor units, each ranging in numbers from tens to thousands, is innervated by a motor neuron. For instance, the biceps working the arm are served by about 774 motor units, each controlling an average of 750 muscle fibers [2]. Amazingly, it is the unsound and unstable structure that is being exploited in bipedal functionality, with capabilities of fast response and agility. We think of stable structures as having a wide base or a low center of gravity, which can settle back to equilibrium under perturbation. However, imagine how unwieldy and cumbersome it would be to ambulate if our base were like a pyramid.

A stick standing on a table falls readily, but on a finger, the hand can move to follow its center of gravity to counter the falling moment and keep it in balance dynamically. Likewise, a stationary bike is unstable, but when it is riding, it stays balanced by the angular momentum of the wheels, without sophisticated control systems, just the steering hand bar. A slight turn changes the bike's direction, creating a torque that counters the gravitational moment, thus keeping it in dynamic balance. We learn to ride a bike by relying on the instinct to steer from the direction of fall, but without having to know the continually varying forces that keep it in balance.

Not unlike an inverted pendulum, our skeletal frame falls readily. In our ambulation we are literally righting the body falling between states of unstable equilibria to keep balance. The mechanism of dynamic balance is preprogrammed in our neural circuitry and monitored by sensory inputs of our vision, inner ear, and proprioception, which occurs without our conscious effort. As we shall see, the spinal engine plays a primary role in our gait.

\section{Anatomy of the Vertebral Column}

The vertebral column does seem unsound, but its anatomy in details gives more comfort. The intervertebral discs (annulus fibrosis) which cushion between the vertebrae can withstand compression much better than the bones [3]. Ligaments securely bind the vertebrae individually to one another and crossing over to others as well, making it robust and flexible. Likewise, deep muscles, which strap along the column, between and crossing over several vertebrae, further strengthen and stabilize the spine and allows for local movements (see Figure 4). Also, the vertebrae increase in size gradually from the neck to the lumbar base, which enhances the structural strength of the column.

Critical to the soundness of the erect structure is the support of the column at the junction of the sacrum and pelvis (ilium), namely, at the sacral-iliac joint (SIJ). The plane surfaces of the SIJ interlock in their irregular grooves and ridges, which secure the lumbar-sacrum support. The ligamentous bindings and muscle strappings are even more elaborate and secure here- -35 muscles attach the sacrum to the hip bones and the ligaments here are the strongest in the body. Thus buttressed at the base, the vertebral column, bound by deep axial muscles and ligaments, enjoys the integrity of strength and stability, and is equipped to withstand the compression, torsion and loads that the body may be subjected to.

Besides the multifidus muscles immediate to the vertebrae and along the column that extend to the sacrum three muscles secure and stabilize the lumbar spine to the pelvis (Figure 5). Attached at the lumbar vertebrae, the psoas muscles cross over the pelvis and the hip joint to insert at the femur head (the psoas minor inserts at the lower pelvic arc); the iliacus attaches at the pelvic crest and joins the psoas major to also insert at the femur head. (Together they are referred to as the iliopsoas, which appears often in the discussion of lower backaches). The quadratus lumborum, laying behind the psoas band, attaches at the lowest rib to secure the spine to the pelvic crest. The primary function of these muscles is to secure and stabilize the lumbar spine and to maintain the integrity of the erect structure. If in an action, this core integrity is violated, then its performance outcome would suffer significantly. Also, if the integrity is not maintained,

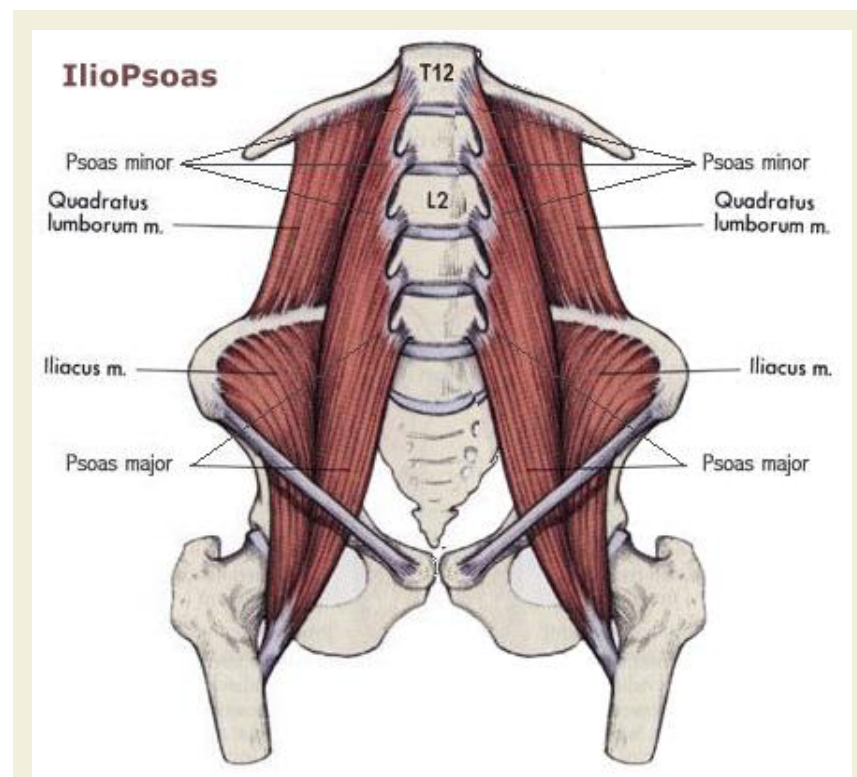

Figure 5: The three muscles that secure the lower spine to the pelvis 


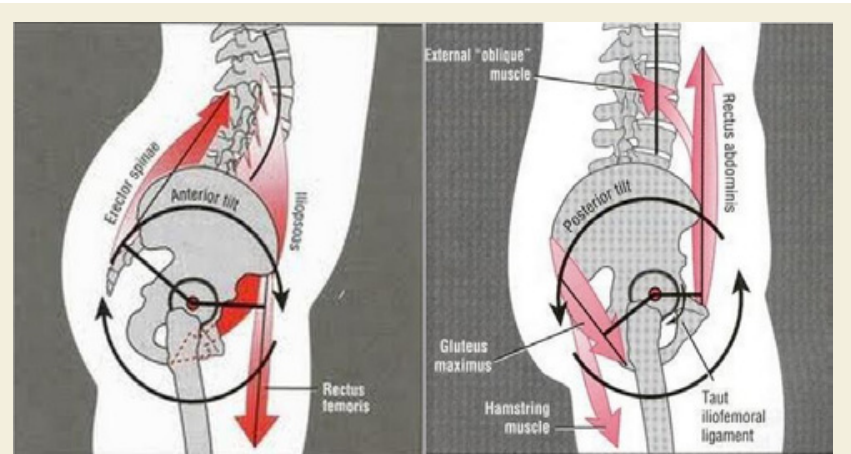

Figure 6: Nutation

Counter-nutation

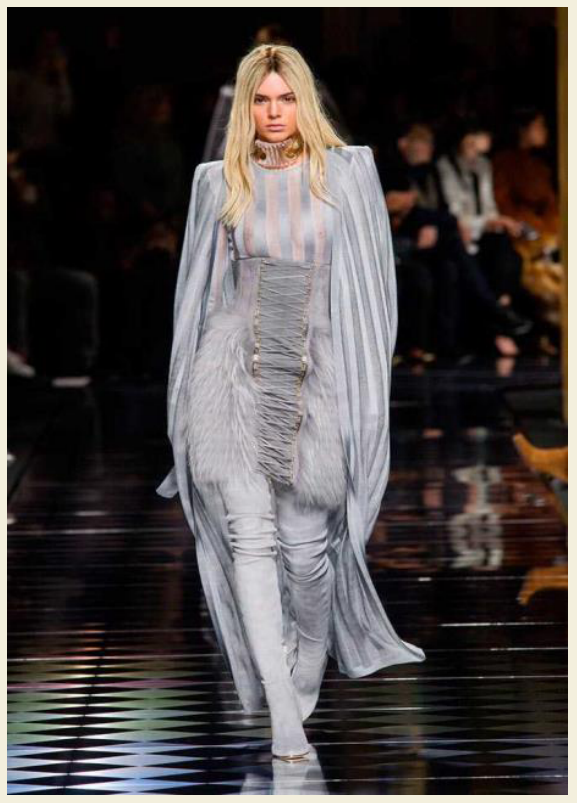

Figure 7: A fashion model's walk.

chronic backaches would result due the persistent stress and strain on the muscles there.

\section{The SIJ Hub}

The movements at the SIJ plane joint are very limited, in an average range of 2 to 4 degrees in each of the three planes. Of significance to spinal curvatures is the larger range of about 4 degrees in the rotational movement of the sacrum in the sagittal plane, "nodding" forward in nutation (tailbone turned backward), or rocking backwards in counter-nutation (tailbone forward), at the transverse axis through the second sacral segment (S2). Lordosis is increased by nutation and decreased by counter-nutation (Figure 6). The rotation in the transverse plane is the axial rotation of the lumbar spine, which taken as a whole includes the incremental turn of each vertebra. The translational movement of the sacrum is limited to about $1.6 \mathrm{~mm}[4]$.

The movement of the spine is linked to the movement of the pelvis at the SIJ. And the movement of the pelvis occurs at the balland-socket hip-joints. Thus, the pelvic platform provides not only support for the spinal column, but is also a conduit of motion and loads between the spine and the femurs, which are described as the three levers by Vleeming [5]. Though the adjustments at the SIJ are small and internal, the SIJ plays a critical role in the transmission of motion and force between the spine and the femurs via the pelvic platform. In fact, the SIJ form the 'hub' of forces transferred from the trunk to the ground and vice versa (Lovejoy, 1988, Aiello\& Dean, 1990) [6].

The principle of the SIJ hub strikes at the core of the art of body motion, which in many respects, is a play of load transfers between the trunk and the legs. The transfer occurs through two interlinked triangular conduits: the smaller lumbar-sacral joint (L5/S1) and sacral iliac joints at the base of the sacrum, and the larger SIJ and hipjoints of the pelvic platform.

To reiterate, the transmission of motion between the torso (spinal column) and the legs (femurs) through the pelvic platform is controlled at the triangle of joints: the SIJ and the pelvic joints. However, the control is complicated by the coupling between the axial torsion and the tilting of the pelvis due to the spinal curvatures-the effects of the spinal engine. Critical in sports, martial arts, or any performance arts is the control to align the sacral and pelvic movements, the precision of which is necessary to harness the full potential of the body. A slightest error at this basic level could amplify in costly degradation of output in performance.

This paper is about the pragmatics of cultivating the hub principle to transfer force and load at the pelvic platform through the methodology of Taijiquan. Taijiquan has long recognized the hub principle at the sacral triangle - the L5/S1 and the SIJ-by the principle of the centrality of the dantian, to be discussed. This turns out to be at the core of the training to harness what is generally called waist power, and here is where the spinal engine plays a critical role.

\section{The Human Gait and the Spinal Engine}

This review is to bring out the spinal engine in the human gait, so that we can appreciate its contrasting role in generating waist power discussed in a subsequent section. We certainly would not argue that we use our legs to walk, but challenging conventional thinking, Gracovetsky argued that walking did not need legs. He put forth the proposition that the role of the spinal engine is primary in our gait. This is dramatically demonstrated by the ischia of the pelvis serving as

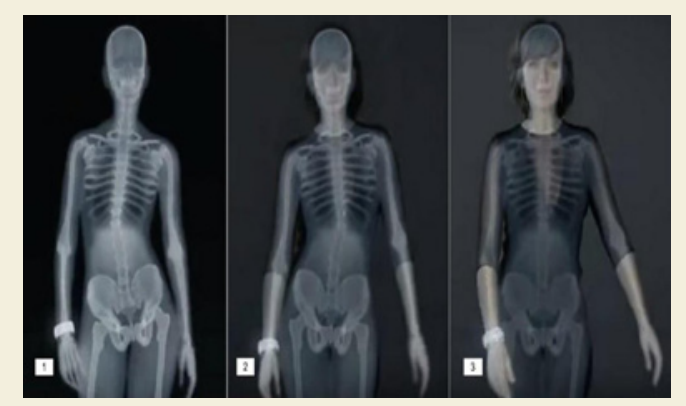

Figure 8: X-ray vision of the spinal engine at work: frame 1 weight on right, stepping forward on left, thoracic rotation to left zeroing out lumbar rotation to right at about T12/L1; frame 2, steps changed; frame 3, spinal curvatures in about same sagittal plane in neutral. 


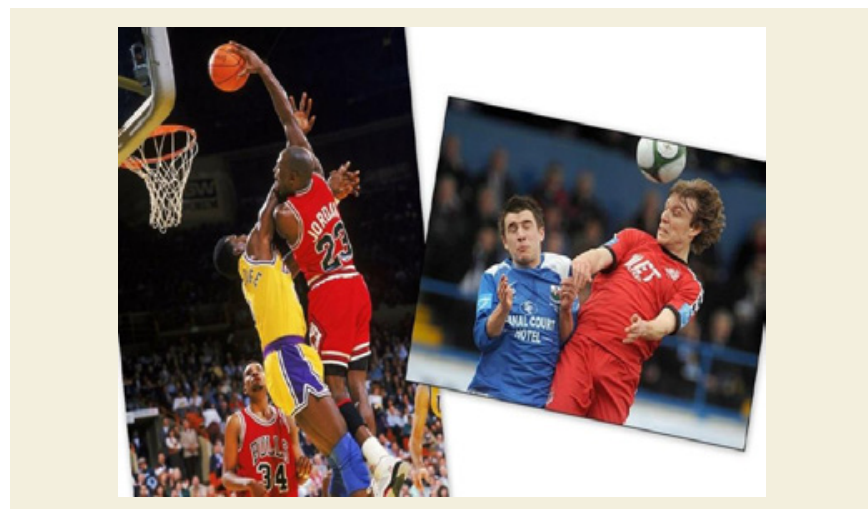

Figure 9: L. Blocked in the air, Michael Jordan dunks the ball R. Wesley Boyle heads the ball in the jump.

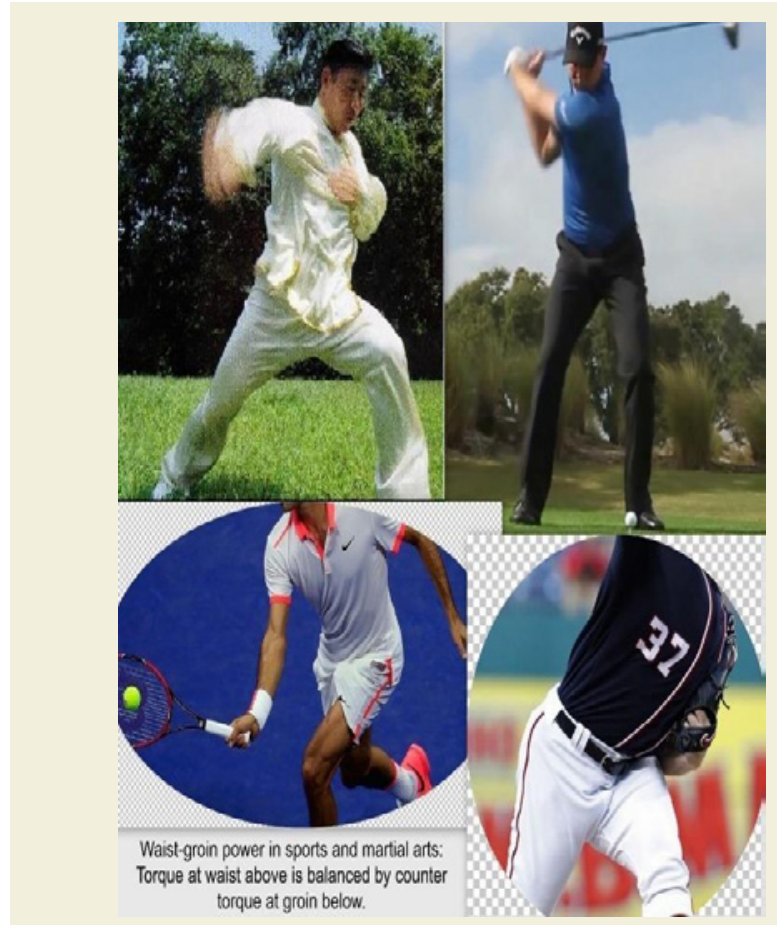

Figure 10: Waist-groin power.

legs in the ambulation of a subject born with no legs [7].

We do not flex and extend our spine like a lizard in locomotion. In our upright posture, the spinal torsion is produced by the flexion in the spine done by the tilting of the pelvis. As we take a step forward, say with the left foot, the pelvis tilts on the weight-bearing right, and the curved lumbar part of the spine bends in the direction of the tilt, while that of the thoracic spine turns in the opposite direction. Thus the waist (lumbar) rotates to the right, while the chest rotates to the left in the walking stride. Similarly, we see the rotations in the reverse directions when we push forward with the right leg. Note that in each stride, the angular momentum of the chest cancels out that of the waist in the walk, as depicted by the arm swinging counter to the stepping.

Still, a good part of the work of walking is done by the leg muscles.
The forward step and stride are propelled by the muscle actions of the legs and ground friction and aided by the forward momentum. The kinetic energy is recycled; it is stored as potential energy when the body's center of mass is raised from when both legs are astride to when they are together. Thus walking is a cycle of converting potential and kinetic energies aided by hip extensors and the stabilization of the body by the dynamic balancing of the lumbar rotation and the counter-rotation of the chest and shoulder, aided by the friction of the foot on the ground. The dynamic zeroing out of the angular momentum reduces significantly the need to expend muscle energy to apply "brakes" to readjust and stabilize the body in motion. This is one reason why our hunter-gatherer forbears could trail down and capture their wild prey by dogged pursuit, a measure of human durability and sustainability in locomotion over the animals [8].

In other words, the spinal column is not a free-loader riding in the carriage of the pelvis, carried by the legs in locomotion. The spinal engine pays more than the torso's fare share-it plays a primary role in our gait. The spinal engine is very evident in the gait of a fashion model. The pelvic tilt is more pronounced as her steps are crossed in the catwalk. With an evocative look of coy indifference, the walk exudes sensual appeal as the spine sways in rhythm to her pelvis rocking side to side (Figure 7). Interestingly, the mechanics of the spinal engine is captured in $\mathrm{x}$-ray vision in a Citracal TV commercial (Figure 8) [9].

\section{Spinal Engine and Dance}

Michael Jackson exploited the spinal engine fully when he rocked his pelvis in the defining moonwalk dance to his song, Billie Jean [10]. The 1982 release became an all-time worldwide sensation. The spinal engine hummed to the tune, which created a harmonious fusion of dance and music. Would the song be the same without the spinal engine running? The spinal engine is of course critical in all forms of dancing, not just in the pop culture. In classical Indian dancing, the head and neck dance movements (shiro bheda and griva bheda) are codified into nine shiro and four griva $[11,12]$. The movements require that the spinal engine be in tune and harmony.

On the other hand, diametrically opposite to the gyrations of the pop-culture genre, the spinal engine is subdued by restraining the pelvic tilts in the Ryukyuan (Okinawan) Dance. The dancer moves by keeping the pelvis from tilting and the center of mass level. The slow

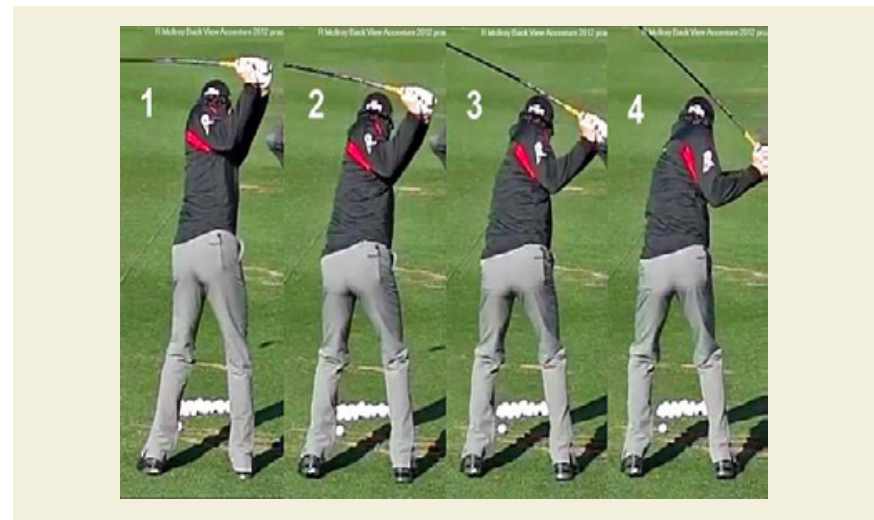

Figure 11: The waist-torque is supported by the groin-torque in Mcllroy's downswing. 


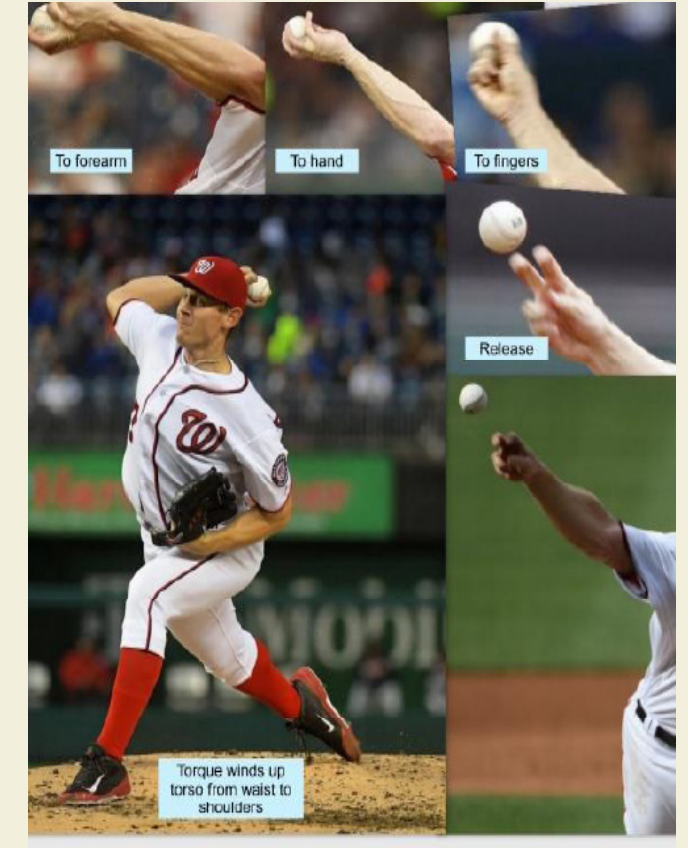

Figure 12: Stephen Strasburg of Washington Nationals by Katherine Frey/ Washington post July 14, 2016.

dance movements are stylized, calm and not strained-the spinal curvatures stay close in the sagittal plane idling the spinal engine [13]. Taijiquan utilizes similar techniques to discipline the spinal engine.

\section{Spinal Engine in Sport Actions}

Unlike dance movements, sports actions are not choreographed, but are characterized by bursts of enormous energy on demand, for instance, to interact timely with an externally directed source, such as a ball. The soccer player running after the ball does not stop but kicks it on the run, or heads the ball on the jump in the air. The basketball player ducking around the guard does not stop but shoots the ball over the block. The pictures of the actions in the air capture the dynamical balance with gravitational moments, and the unison of the body's rotational moment, which require harmonization with the spinal engine (Figure 9).

These sport actions are very much focused on the ball-in the hoop or goal-and they require responses of the spinal mechanisms that are more endowed than trained. That is, a weekend warrior cannot hope to train to improve much the skills of juking or dodging like a professional player. In golf, tennis and baseball, the explosive power behind a long drive, a forehand swing, or a fastball itself is of great fascination. What is the biomechanics of the swinging power and how can one train to improve on it significantly even if one is a recreational player not endowed with athletic talent? This brings us to the source of the body's core power, generated at the waist-groin region.

\section{Waist-Groin Power Dang-Yao Jin}

We admire the explosive power of a golf swing, but the golf ball at the tee sees only the momentum of the golf club. The force that sends the ball off is a consequence of the physics of collision between the ball and the club head. How long the drive will be depends on the momentum of the club transferred from the momentum of the body. The greater the momentum the body can generate, the greater the impact force of the club.

The different segments body must move in unison for the momentum to unify, and the most crucial component is the trunk's rotational motion. This is not about the body spinning in a pirouette, but harnessing the trunk's muscles to maximize its angular momentum with control of balance. In the rotational motion, the upper body turning in one direction must be balanced and supported by the momentum of the lower body turning in the opposite direction. Where should the division of rotation be between the upper and lower body?

The distribution of the body's mass between the upper and lower body is most proportionately divided at the waist-groin junction. This means that the greatest angular momentum will be generated if the rotation occurs at the waist-groin junction, hence the term waistgroin power or dang-yao jin 裆腰劲. Taijiquan refers to this junction as the kua.

If the upper-body rotation occurred at the chest level, then the muscle mass of the midsection would not be contributing to the upper body's rotational momentum. If the upper body turned at the junction of the knees, there would not be enough muscle mass below to stably support the overloaded action, which would degrade control and balance and strain the knees and ankles, causing injury. Thus, we see the rotation and counter rotation occurring at the waistgroin junction in the sport actions captured in Figure 10. In each of the right-handed action, the punch, downswing, forehand stroke, or fastball, the torso's rotation to the left above the waist-groin junction is mutually supported by the counter rotation to the right of the base below the junction. Note that "the groin is hooked inward" (kou dang 扣禅) on the left side in counter-rotation in support, and is aided by the friction on the ground.

\section{Waist-Groin Power in the Golf Swing}

Let us review the golf swing to see more clearly the biomechanics of the torso turning as a whole at the waist-groin junction. The torso's rotation in Rory McIlroy's downswing is captured in Figure 11. In frame 1 , the torso is wound up about 90 degrees to the right, coming to the end of the upswing phase. The weight is more substantial on the right. This upswing rotation is a composition of the rotations of the pelvis, the SIJ, the lumbar and thoracic vertebrae, each turning a few degrees incrementally up the spine in the same orientation. In frame 2 , the weight is shifted to the left and the left foot is anchoring to begin the downswing phase, and the swing begins to "unwind." (This shift in weight is in conjunction with the "shoulder drop," as observed by Kelvin Miyahira [14]).

In the downswing rotation to the left (frames 2 through 4 ), the torso mass and muscle actions are unified in angular momentumthe back remains intact throughout and the torso turns as a whole. The rotation occurs at the pelvic junction, driven at the triangle of joints: the SIJ and the hip-joints. The pelvis, supported at the balland-socket joints, provides the reaction force for the left groin to "hook inward" and the ground friction to form the counter rotation 
(of the lower body) to balance the swinging action. The rotation at the pelvic junction and the whole muscle-mass of the torso is captured in the video clip http://imgur.com/SYA95Sp.

However, getting the muscle-mass of the torso to turn as a whole would be a departure from its customary mode of rotation in our gait, which habituates us to turn the upper body at around the thoraciclumbar vertebrae T12/L1. So we tend to do the same in the waistpower execution, turning at the chest level, which would then deprive the action of the muscle power of the midsection, a serious flaw that would cost a hundred plus yards in the drive. To turn with unified momentum, the spinal torsion must be harmonized with the torso's rotation. So the physics part is easy. The hard part is to get the body to do it. Let us review the muscles of the waist-groin region from a functional perspective.

\section{Functional Perspective of Muscle Actions}

Reviewing the muscles of the waist-groin junction, we find the long muscles that attach at the lower pelvic ischial base, running along the femur, to the heads of the tibia or fibula (the biceps femoris, semitendinous, semimembranosus), and the longest one, the sartorius, which extends across obliquely from the iliac crest to the head of the fibula. These muscles produce torque in the hip rotation, but the body is more familiar with the flexion and extension and the adduction and abduction [15]. A good part of the torque turning the hips comes from the frictional force at the feet, negotiated through movements at the knees and ankles, and the internal movements at the hip-joints and SIJ.

To generate maximal waist-groin power, all these torque actions from the long to small to deep muscles, particularly those at the SIJ and hip-joints, must align and balance and be in tune with the core axial muscles of the vertebral column. Any misalignment would impair power output. Besides torque, there is another functional perspective-the kinetic line-up of the muscle actions. Look at the action of a punch. In the anxiety to throw a fast punch, the muscles closest to the punch action, namely, the prime-mover muscles of the arm and shoulder would tend to dominate, causing the fist to jump ahead in the action. This would cut the muscle power and momentum of the other parts of the body from contributing to the punch, lessening force. That is, the muscle actions must align in sequential kinetics.

Indeed, it is very common for prime-mover muscles to dominate in function. Our neurobiology is not concerned with muscle actions jumping out of kinetic sequence as long as the job gets done. To overcome the problem in training, Taijiquan divides the skeletal muscles into two functional groups:

- Outer or prime-mover muscles that move the body segments, such as the arms and legs. These produce the body's translational as well as rotational motion, thus include the muscles involved in producing the torque or moment.

- Inner or deep muscles that secure the joints and maintain the integrity of the skeletal structure, including the core axial muscles of the spinal column.

The muscles are not exclusive in the groupings, neither are they dedicated in their functional roles like the smooth muscles. As we shall see, training to generate body motion with control and unified momentum is an interplay of the inner and outer muscles, and of the torso's torque muscles and the core axial muscles, to balance and align in functional accord-the principle of "inner balance". The principle guides to sort out the muscles competing in their functional roles. It should be noted that conventional synergists and agonistantagonist muscle pairings are subsumed. Next, we wade into the waters of Taijiquan in the study of "inner balance".

\section{Inner Balance}

Taijiquan's slow-motion methodology does not just develop dang yao jin or waist power, but offers an uncanny solution to training strength, not just any strength, but extraordinary strength, called neijin 内劲 (“Internal Strength"). This pronouncement usually comes as a surprise as the slow-motion exercise cannot be more remote from the power crunching exercises of strength-building. To take a little of the mystery away, think of the quaint and unorthodox training as regulating body motion to be in accord with the Taiji theory of yin and yang. Then with a developed store of neijin, the body's response in combat, imbued with the grandness of Taiji theory, becomes both an offense that is unstoppable and a defense that is impenetrable. That is why kungfu peers have always regarded Taijiquan as a martial arts of the highest order [16].

Here is an illustration of balance that Taijiquan is concerned with. We take a deep breath in a medical checkup as directed by a physician with a stethoscope placed on our chest. In so doing, we inadvertently raise our ribcage and hollow our abdomen. This tightens the back muscles and weakens the internal structure of balance, rendering the body top heavy and easy to topple. The posture is of weaker balance. The illustration shows that underlying each physical function there are varying combinations of hundreds of muscles that can support the same task. In sports, picking the right combinations is crucial to better performance results, while in mundane activities, the combinations are less important as long as the job gets done. What are the preferred combinations of muscle actions? Taijiquan training picks a preferred combination of the muscle actions as that which corresponds to a state of better yin-yang balance, or simply that of better balance.

A state of inner balance is defined as one that conforms to yinyang balance. Manifested in biomechanics, inner balance is a state where the muscle actions at the joints are neither excessive (too yang) nor deficient (too yin). Inner balance encompasses the full comprehensiveness of balance in bipedal functionality under all situations between the forces of muscle actions themselves and with gravity and other loads on the body. Taijiquan practice is all about resolving muscle actions to infuse the body with the principle of inner balance.

The key to the practice is the cultivation of the senses to discern what is excessive or deficient in muscle actions and the development of the tools to resolve them. The insight of the practice is simply to discern the errors and to keep chipping them away. This puts one on a pragmatic path of practice that leads to inner balance by reducing the margin of errors, without the burden of having to know the complex of muscle activations. And in the process, the body's core strength develops and is that of neijin. 
The import is that inspired by the principle of inner balance, the response of neijin is governed by balance and is lively and agile in the interchange between yin and yang. So the body segments can be maneuvered with ease to align in sequential kinetics for maximizing momentum. The payoff is that the force that ensues from motion regulated by the principle inner balance is consummate. And of course, ever present is balance, which is basic in bipedal functionality, without which there is no control.

However, we cannot regulate our movements to comply with inner balance by allocating so much muscle actions here and so much there, as we do in adjusting weights in a balance scale. The muscle activations are just too complex and the neural-motor processes are not wired to do that. Interestingly, we find, in the unorthodox slowmotion methodology of Taijiquan, a tool to resolve the errors-the tool of "fangsong." But before we get to it, we need a brief review of the neural-muscular activities.

\section{Neural Wiring and Responses}

A basic issue is that our neuromuscular system does not pay heed to the principle of inner balance. If it did, then fastballs, long-range drives, and neijin would be common place. Quite to the contrary, the neural responses to a command often elicit muscle activations that can frustrate the task at hand.

For example, in the mundane task of picking up a box, we bend forward, out of convenience, to reach for it. The back muscles fire up by reflex to keep the leaning posture from falling over. In lifting the box, the weight pulls the body further down, triggering more muscle activations to keep balance, and even more to raise the load, resulting in much reduced muscle energy left to do the work.

By stepping closer to the box and bending at the knees, the posture is more balanced internally and the task can be performed with better leverage and the help of leg muscles, thus less effort. The second posture is in a better state of inner balance. But the neural responses do not attend to postural factors of balance. Not only that, the neuronal firings of the back muscles to keep the immediate balance, exacerbate the poor leaning structure, the cause of many a lower back ache. We are presumed to have control over our voluntary movements, but the control we have is limited at the top hierarchy of the motor chain, in the command, say to touch the nose. The simple command triggers a complex of neural activities and a flow of signals and information data between the brain and muscles, which are completely opaque to us. We only know that the muscle activations result in the movement-output of the hand touching the nose.

The limited control we have of our somatosensory muscle system-the volitional part-is very limited indeed. We cannot execute a plan of muscle allocation according to an engineering solution to comply with inner balance even if one could be devised. We cannot tell which muscles are wanting in a golf drive or a punch. That being said, the neural wirings of our musculoskeletal system give us a remarkable range of versatility in dance and sports and the finest of control of our hands, as attested to by human artistic endeavors.

The problem is that we have no sensory feedback of the overall structure of the body on the fly with regard to the relative state of muscle actions as to which combination is better or preferred. The issue may or may not be pressing for routine activities, but still relevant to health-to prevent chronic back aches. However, the issue is critical for generating the strength demanded in performance. This is the source of frustration in training-we cannot summon the body to execute the movements with the accuracy and power we wish, even with dedication of practice, as weekend golfers know well.

The neural responses for a given action do not activate the same but varying combinations of muscles. The activations are influenced by habits of convenience and formed and evolved by preprogrammed elementary pattern motion-generators. The neural responses do not attend to inner balance, and often undermine the principle. In other words, often, the "natural" responses turn out to be barriers of neurobiology to training in sports and martial arts. Unless addressed, they persist as inhibiting factors that stymie training-the syndrome of hitting-the-wall.

Between the commands of action at the top hierarchy to the motor neurons activating the muscles at the bottom, there is a huge gap of neural activities that we have no cognition of. There is no feedback of the execution as it is occurring. By the time the result can be gauged as good or bad, it would be too late, especially, in a combat situation where the target is also moving. How do we bridge this gap, which is critical to the discipline of muscle actions? How can we resolve the muscle activations that we only have limited or no cognition of, and often are themselves the result of the responses of neurobiology? Taijiquan's solution to this conundrum is the prescription of fangsong, a tool to resolve the yin-yang errors of muscle actions.

\section{Fangsong Relaxation and $Q i$}

To illustrate the concept of fangsong, hold an arm out to the side; it is in physical balance between its weight and the muscle forces supporting it. To better discern the balance, stretch the hand out as much as you can. The stretching increases the muscle activations, which is sensed as a tensing up of the arm, and is changing the internal dynamics of the muscle actions in support of the balance. Holding it in position for a duration, discomfort of stiffness would set in, a consequence of tenseness of the muscle actions, a discerning that balance can be better. Upon sensing the discomfort, the reflex is to relax or let go to lessen the tenseness. The reflex response has the effect of resettling the muscle actions to one of lesser discomfort in the physical balance-a better balance of the internal structure. This response represents the rudiments of the tool of fangsong 放松 which translates as "relax and let go."

However, the state of muscle interactions cannot be readily sensed, as when in motion. This is where the slow-motion methodology comes in-it acts to moderate muscle actions that tend to dominate. More ingeniously, the practice is guided by the constant admonishment of “using mind-intent, not force” (yong yi bu yong li 用意不用力). The mantra is baffling as "not to use force" is often loosely translated as "not to use muscle-force," which makes no sense as movements are produced by the contractile forces of muscles.

As it turns out, the cryptic mantra is equipping the fangsong tool with two prongs to work on the muscle actions. The first is the mantra's urging of "not using force," which in the deliberative slow-motion imposes a discipline that forestalls and subdues the outer muscles from dominating in the action. The second part of the 
mantra of "using mind-intent" gives time for the deeper and inner muscles to activate to stabilize the structure at the core. Both work to prevent the appendicular muscles and the torque muscles from jumping ahead of the axial muscles, and thus for them to better align and balance. Therefore, the practice mantra in slow-motion is persistently tempering the outer and prime-moving muscles, and pumping the inner and axial muscles in their crucial roles to secure, stabilize and balance support at the joints and the vertebral column, especially at the SIJ.

Because fangsong requires attentiveness to the process, the body becomes aware of the joints. Also in the process, the body gains sensation of the bioenergy associated with an ease of motion flow at the joints. This bioenergy is identified as $q i$ 气 the life-force energy of Traditional Chinese Medicine (TCM). This qi grows with the fangsong practice.

The qi nurtured is associated with the fangsong resolution of the muscle actions-the better the balance, the more the qi. In time, as $q i$ grows, fangsong relies on $q i$ as a medium to discern and resolve imbalances. The practice becomes more internal, with the focus of fangsong shifting more to $q i$ flow. So impedance in $q i$ flow is deciphered as imbalances. By its operation, fangsong cultivates a dynamics of $q i$ through the joints, which is internal, in contrast to motion which is external. And importantly, the cultivated $q i$, in time, becomes viewed as qi dynamics regulating the motion. In other words, qi serves as a marker of sensation associated with fangsong to gauge the resolution of muscle actions towards inner balance.

There is another dimension to the fangsong tool, which incorporates a complementary function to strengthen support that is languid. Stretching a droopy arm may add strength to the support, but it also tenses up the arm. To do this without tensing, the fangsong stretch is "internal," described as "stretching the tendons and bone" (shen jin ba gu 伸筋拔骨). The internal stretching gives a connectivity of energy to the motion, thus strengthening the lax support, analogous to giving tautness to a slack string, more of which is discussed in the author's paper, Generating Body Strength Through Taijiquan Motion [17].

Thus the fangsong tool itself is dual-pronged in operation: one by fangsong-relaxing when it senses tenseness and the other, by fangsong-stretching internally when it feels laxness. Working side by side, it keeps the support in the goldilocks zone-neither too tense (yang) nor too lax (yin) and with it the associated qi.

The Taiji strategy is to stay in between the excesses or deficiencies of yin and yang-in the middle ground so to speak, and to work on reducing the margin of errors. The margin of errors tapers by the sharpening of the organic fangsong tools and the heightening of the discernment, which ushers the practice path in convergence towards inner balance. And this is induced by the discipline of meditation - the mind part of the practice, which develops by the constant attentiveness to the process. In this sense, one is not directly striving for inner balance, only resolving the errors of imbalance, a sort of "doing without doing" (wei wu wei 为无为). The path of staying in the middle ground exemplifies the quintessential way of the ancient philosophy of Dao 道.

The development of qi energy in practice is gradually but crucially bridging the gap of neural activities, leading to the realization of the $y i$ $q i$-motion paradigm: The command of $y i$ 意 (mind-intent) activates $q i$, and $q i$ signals to innervate muscles, and thus the $q i$ arriving drives the motion. Since $q i$ energy is cultivated, we are conscious of it. This gives the body a sensation and feedback of motion at the joints through the qi medium, thus bridging the gap of neural activities. In other words, $q i$ serves as the internal dynamics associated with the smooth transmission of motion at the joints. And as mastery matures, $q i$ dynamics becomes more unified with motion, which is a representation of the principle of inner balance.

\section{A Basis of Qi in Taijiquan}

The "life-force energy" $q i$ is taken as given by TCM, but is viewed as a bioenergy sensed in association with fangsong resolution. The experience of $q i$ as a somatosensation serves the purpose of this paper in terms of cultivating inner balance and generating strength. It relies on the sensory data from the muscle spindles (of muscle stretch and speed of stretch), and the golgi tendon organs (of muscletendon tension). In composite, the senses stimulated by tension and movements of muscles and tendons at the joints (kinesthesia), of position and space (proprioception), of balance (vision and inner ear), of pressure, pain and temperature, and light, as well as of blood flow, are viewed as qi-sensation.

Changes of physiology and bioenergy that arise from fangsong practice have been studied at the Laboratory for Mind-Body Signaling \& Energy Research and Susan Samueli Center for Integrative Medicine, University of California, Irvine. The research team headed by Shin Lin has quantified increases in the following physiological and bioenergetics changes due to Taijiquan or qi-energetic exercises: a) blood flow (perfusion), b) state of relaxation as indicated by heartrate variability and brain-wave analysis, and c) bioenergy emission in the form of heat, light (photon counts), electrical charge, and conductance at acupuncture points [18].

These changes of bioenergy are qi surrogates but are sensed differently. Blood perfusion, experienced as tingling sensation in the hands, is most prevalent in qigong practice, while photon count is not sensed as it is small, though the increase is significant. Much of the $q i$-sensations in Taijiquan are changes in bioenergy that stimulate the sensory receptors in the tendons, muscles, bones, and fascia, most notably, by higher activation levels of muscles. Together, they offer an experimental basis to the experiential knowledge of qi dynamics in Taijiquan.

In the fangsong play at the SIJ and pelvic joints, the activation level of the quadriceps muscles is raised 2 to 4 times more than in walking [19]. The higher activation levels are registered as a $q i$ sensation. Muscle activations in walking rely primarily on neural pattern generators, but fangsong induces the muscles to settle more into the joints. In the deliberative slow-motion, the outer and primemoving muscles that tend to dominate are restrained so that the inner muscles get to fire more. The higher activation level of the inner muscles is often sensed as a surge of heat and as qi.

Taijiquan, of course, has long validated the basis of $q i$ in the timehonored wondrous kungfu of neijin. Neijin arises from motion that is harmonized with $q i$ dynamics. That is, in Taijiquan, $q i$ is grounded in the context of neijin. The qi dynamics (internal) regulating motion 
(external) represents the "unity of the internal and external" (nei wai jie he 内外结合).

In traditional Taijiquan theory, the fruition of this unification of qi is borne of the three internal unities of: heart (xin 心) and mind (yi 意); qi 气 and force (li 力); and tendons-muscles (jin 筋) and bones (gu 骨).

The conditions for the heart and mind to be one are nurtured by the meditation component of the practice. The attentiveness in the slow-motion practice inculcates awareness that tempers the worrying and distracting thoughts flitting in and out, quiets the mind and clears the murkiness. The unity of the heart and mind sharpens perceptiveness and deepens awareness for insight to arise. With perceptiveness heightened, the fangsong resolution can better activate the deep and inner muscles, as well as the axial muscles, which secure and stabilize the joints and the spinal column. This is the discipline of "uniting" the tendons-muscles and bones. The unities of the "heart and mind" and "muscles and bones" are conditions for $q i$ and motion (force) to unify to give rise to neijin.

\section{Dantian Centrality and SIJ Hub}

The task of checking the balances at the multitudes of joints one by one is clearly a daunting task. But what makes it more daunting is that resolving the errors at one joint may affect that of the other joints because of the skeletal structure's tensile integrity. This means that the resolution at one joint requires a recalibration at the other joints each time, which would make the undertaking nigh impossible.

To compound the task even more, each body segment has its own power plant of muscles. This makes the problem of resolving the muscle actions of the segments to align and balance, analytically intractable. Amazingly, guided by The Ten Essential Principles, the problem is reduced elegantly to a principle of centrality [20]. The centrality flows from the eminent role of the waist-groin region as the source power. Commonly called the waist, the Taijiquan parlance for it is specific, namely, the kua 胯, which refers to the complex of the pelvic platform and the triangle of joints and their functional play. Kua is inextricably linked to the rotation of the torso and the transference of force between the three levers-the spine and the legs.

The balance cultivated by fangsong at the kua and the triangle of joints is fundamental and forms the foundation. From this kua base, fangsong is extended to work on the corresponding pair of joints of the shoulder and $k u a$, then to the elbows-knees and the hands-feet pairs. The midpoint of the kua turns out to coincide functionally with the energy-point of Qi-energetics, called the dantian. Picking the dantian as the center of reference provides a refinement of fangsong of all the body's joints. And by referencing to the dantian, the fangong reinforces the core balance, and more importantly, nurtures a centrality principle at the point. The dantian 丹田 ("field of elixir") is located at three fingers below the navel and about a third of the way inside. The navel is positioned at about the vertebral level of L3/L4 with slight variations [21]. The relative measure of the width of three fingers puts the dantian at the approximate level of the SIJ [22].

The dantian is not just a reference point in name. By referencing to the dantian, the fangsong is actually tempering and working each time to resolve the muscle actions at the triangle of joints, which thus is actually developing the centrality of the dantian. Also, crucially, the $q i$ cultivated in the fangsong resolution at each joint relative to the dantian is nurturing a connectivity between the joint and the dantian, forged by inner balance. This builds a web of qi-connectivity of the joints centered at the dantian, which forms the basis of the principle of dantian centrality that alleviates the sticky problem of the matrix of joints.

The body's comprehension of the centrality principle is reinforced by $q i$ cultivated in the systematic fangsong resolution at the joints relative to the dantian. The constant attentiveness to the dantian and particularly, the fangsong at the SIJ and hip-joints, induce the qienergy to collect in the lower abdominal region, concentrating at the dantian. In time, the nurtured concentration of $q i$ culminates as the “fullness of dantian qi" (dantian qi baoman 丹田气饱满). The fullness of dantian qi gives the body a deepest experiential appreciation of the centrality principle.

The dantian $q i$ may seem esoteric, but it works indispensably to resolve imbalances at the deepest vestiges, cutting the Gordian knot of inner imbalances, that leads to its maturing fullness. With this achievement, the body is said to be invested with the principle of centrality. The significance of the investiture of the central status on the dantian (yi dantian wei hexin 以丹田为核心) is that in the $y i$-qi-motion paradigm, motion is directed and regulated by dantian $q i$. The corollary is that the motion directed by dantian $q i$ is regulated by yin-yang balance and forged by inner balance. Therefore, the motion (thus momentum) transmits between the upper and lower body through the dantian center in balance and alignment with ease, without obstruction. This means that the dantian is serving as the hub of forces transferred from the trunk to the ground and vice versa without loss of energy due to flaws of inner imbalance. Dantian centrality is the realization of the hub principle of the SIJ.

Dantian centrality is the piece de resistance in the theory of Taijiquan. Grandmaster Chen Xiaowang, $19^{\text {th }}$ generation descendant of Chen Family Taijiquan, has been instrumental in the articulation and propagation of this principle. To sum up, the attainment of dantian centrality represents the maturity of inner balance and the mastery of the art. Infused with the principle, the body becomes very conversant with the movements at the joints, so can undergo change spontaneously in response with ease and at will according to the loads. Thus, Taijiquan's kungfu maneuvers appear "soft," in contrast to the strenuous exertion of strength associated with brute force. Taiji kungfu is the stuff of neijin born of inner balance [23].

It should be emphasized that the investiture of dantian centrality can only be earned through the long and arduous process of fangsong practice. It cannot be endowed. True Taijiquan masters are made not born.

\section{Harmonizing the Spinal Engine Redux}

Harmonizing the spinal engine with the torso's rotation at the $k u a$ in generating waist power does not in any way alter one's gait of course. In waist-power action, the lumbar and thoracic rotations are aligned in the same orientation so that their momenta unify in the torso's rotation, but in walking, they revert back to turning in opposite directions to zero out. However, it should be emphasized again that the role of the spinal engine is critical in the harmonization 
of spinal torsion, hip-rotation and trunk rotation to produce maximal waist-groin power and in the transference of force between the trunk and the ground. Indeed, the harmonization gets into the deepest levels of practice and rests on the fangsong resolution at the triangle of joints that cultivates the inner balance of the $k u a$, which culminates in the development full dantian $q i$.

More than waist-groin power, this attainment of dantian centrality means that the body is instilled with inner balance and thus is endowed with neijin. Note that the dimension of the body's rotational motions is incorporated in the training by the "silk-reeling" exercise (chansi gong 缠丝功). The silk-reeling trains the kua in leading the series of the body's rotational motions. This engenders the silk-reeling energy, which forms the basis of Taijiquan motion [24].

\section{Taijiquan Training in Sports}

Training regimens may differ in sports, but three basic elements are essential, without which performance can suffer. The first is functional balance to maintain control, the second, the body's core strength, and the third, the body awareness and comprehension of motion at the joints, which is the basis of maneuverability and fine control. These basic elements are at the heart of Taijiquan training of inner balance. This is good reason enough to port the fangsong methodology to sports training. The greater benefit would be to break the barriers of neurobiology - the neural responses of habits and convenience that stymies progress in training and the greatest would be the development of qi pathways to bridge the gap of neural activities to better discipline muscle actions. There have been many anecdotal reports from students that Taijiquan training has greatly improved their golf or tennis swings.

Let us look at the pitching action to bring out one more time the commonality between sports and Taijiquan in the application of waist-groin power. The core power of the waist is transmitted up the torso to the shoulder, then through the elbow, wrist and fingers in the sequential order. The principle of sequential kinetics is captured in the collage of pictures of the pitching action (Figure 12). The pure power of the pitch that delivers a 90 plus mph fastball transmits through the body segments in sequential kinetics from the kua. The torque action at the waist turning to the left (above the kua junction) is balanced and supported by the torque reaction at the groin turning to the right (below the kua). The main picture shows the torque winding up the torso to the shoulders, ready to transfer to the arm. The other pictures show the transmission through the elbow to the forearm, to the wrist and finally to the fingers to power the release of the ball in the sequential order. Any break in the sequence would impair the power output of the pitch.

While it is well known and acknowledged in Chinese Martial Arts that neijin is the stuff that delivers the mesmerizing kungfu feats of Taijiquan, little of it is applied outside of combat. Infused with the principle of inner balance that inspires solidity of balance and the spontaneity in the interchange between yin and yang, the body is rendered soft and hard as needed to meet the unpredictable situations in any physical interactions. Sports, such as football or rugby, can certainly draw on such a lively response of strength, versatility and balance. Imagine a running back dodging his way, unstoppable, against a line of burly defenders, to the end zone with Taijiquan skills! That scene might seem far-fetched, but so would the adoption of the ancient Chinese massage therapy of cupping by a modern elite athlete. Indeed, in the recent Rio Olympics 2016, handsome cupping marks were conspicuous on the bare back of Michael Phelps.

\section{Conclusion}

Even though the paper does not tout the health benefits of Taijiquan practice, the message of its efficacy in health is ever present in the thrust of inner balance. TCM views an excess of yin or yang or a blockage of $q i$ flow as a marker that portends ill health. Thus, the fangsong practice of resolving yin-yang imbalances and developing $q i$ represents the very tenet of health, thus a bargain of preventive medicine by TCM.

Taijiquan's inner balance, as a product of yin-yang balance, also promotes the functional harmony of the internal organs, which are classified under the Five Elements (in parenthesis): liver (Wood), heart (Fire), spleen (Earth), lungs (Metal), and kidneys (Water). The theory refers to the practice as nurturing the internal unities between the organs under the involuntary control of the autonomic nervous system and the skeletal and body parts under the voluntary control of the somatic nervous system: between heart and eyes, spleen and flesh, lungs and body-form, kidneys and bones, and liver and tendonsmuscles. The practice of inner balance covers the unities of the correspondences to attain the oneness of the body:

\section{ZZong zhi 总之 \\ Yi dong er wu bu dong 一动而无不动 \\ Yi he er wu bu he 一合而无不合 \\ Wu zang bai gu xi zai ji zhong yi 五脏百骸悉在其中矣}

In summary,

Once in motion, no part is not in motion

Once in unity, no part is not in unity

The five organs and the hundred bones harmonizing in function.

In this regard, the principle of inner balance asserts the functional harmony of the "five organs and hundred bones" through $q i$ and blood. Thus, the achievement of dantian centrality is also a TCM passport in the passage to longevity. This is saying that inner balance is maintaining a stable environment for the functional harmony of the internal organs, namely, the homeostasis of the biological and physiological environment of the body, on which health rides.

The feature of Taijiquan practice that arguably provides the greatest impact on health and wellbeing comes from the meditation component. Although the practice may begin as a physical activity, the modus operandi of deliberative slow motion entails attentiveness, which grows into meditation that complements the training. The attentiveness keeps the mind from wandering and acts to restrain the "monkey mind" of restless thoughts darting in and out incessantly. Meditation calms and quiets the mind so that it becomes more perceptive of the practice. Meditation thus sharpens the mind, which is necessary at the higher levels of practice where the errors of imbalance are more subtle, requiring more refined tools to resolve. With awareness and tranquility, equanimity takes root, leading to the 
insight of the art-and along with the development, the tremendous health benefits that inure [25].

To sum up, the achievement of inner balance bears three gems:

- Neijin. Body is always in balance, and in response is ever ready to change at will under pressure or load, and the force that ensues is that of neijin and is consummate.

- Equanimity. The keen awareness of the mind keeps at bay, unwholesome and negative responses driven by greed, hatred, jealousy, anger, or more generally, delusions. Thus tranquility and blissfulness are nurtured.

- Homeostasis. The oneness of the "five organs and hundred bones" is the maintenance of a stable environment for the functional harmony of the internal organs.

\section{References}

1. Gracovetsky SA (1989) Spinal engine theory. Springer-Verlag, New York USA.

2. Floeter MK (2010) Structure and function of muscle fibers and motor units: the scientific basis of muscle disease. In: Karpati G, Hilton-Jones D, Bushby K, Griggs RC (Eds) Disorders of voluntary muscle ( $8^{\text {th }}$ edn), Cambridge University Press, India, pp. 1-10.

3. Ibid 1. Gracovetsky cited the 1960s experiments conducted at the biomechanics laboratory of the Wright Patterson Air Force Base in Ohio, which subjected monkeys strapped to a chair to impact forces of 120g's The monkeys shrank by about half as their vertebras were crushed, but their annulus fibrosus remained essentially intact.

4. Arthrology of the pelvis nutation and counternutation childbrith.

5. Vleeming A, Schuenke MD, Masi AT, Carreiro JE, Danneels L, et al. (2012) The sacroiliac joint: an overview of its anatomy, function and potential clinical implications. J Anat 221: 537-567.

6. Ibid 5 .

7. Gracovestsky S (2012) Non invasive assessment of spinal function.

8. Carrier DR, Kapoor AK, Kimura T, Nickels MK, Satwanti, et al. (1984) The energetic paradox of human running and hominid evolution. Curr Anthropol 4: $483-495$

9. https://www.youtube.com/watch?v=8NzPQJJYHRQ\%20.

10. Michael jackson billie jean.

11. Neck movements or greeva bheda in bharatanatyam.

12. Bharatanatyam lessons-Bhedas: movements of the Angas, Upangas and Pratyangas.

13. The ryuyuan dance.

14. http://kelvinmiyahira.com/old/assets/articles/2010/04/2004/images/lb1.gif

15. Ehraheim N (2014) Anatomy of movement of the hip.

16. Ong CP (2017) A Scientific perspective of Neijin (internal strength). Int $J$ Complement Alternat Med 5: 1-10.

17. Ong CP (2016) Generating body strength through taijiquan motion. Int $J$ Complement Alternat Med 3: 1-10

18. Lin S (2007) Research leading to systems/cellular/molecular model for the benefits of Qigong and Tai Chi on health and healing. Scientific and skill papers on Qigong, The World Qigong Forum 2007 and $10^{\text {th }}$ World Congress on Qigong and Traditional Chinese Medicine, Tokyo, Japan, pp. 3-8.

19. Lin S (2013) Electromyographic study on Tai Chi's activation of muscles essential for fall prevention and weightlifting. J Altern Complement Med 19: A30-A31.

20. Chen Zhenglei (1998) Chen Family Taijiquan Theory, Xi'an Science Technical Publication. Chen Changxing's essay, Ten Essential Principles represents the mainstay of Chen Family Taijiquan theory.

21. Ellis $H$ (2006) Clinical anatomy: applied anatomy for students and junior doctors (11 ${ }^{\text {th }}$ edn). Blackwell Publishing, USA

22. Nuket GM, Karabekir H, Ertekin T, Edizer M, Canan Y, et al. (2010) Evaluation of lumbar vertebral body and disc: a stereological morphometric study. Int J Morpho 28 : 841-847

23. Ong CP (2016) The Central Status of the Dantian. The International Magazine of Tai Chi Chuan, Spring 2016 Vol. 40 No. 1. www.Tai-Chi.com

24. Ong CP (2013) Taijiquan: cultivating inner strength. Bagua Press, USA pp. 285 .

25. Ibid 24. Meditation and Perceptiveness. p. 266 